\title{
IN SEARCH OF MARKERS USEFUL FOR EVALUATION OF GRAFT PATENCY - MOLECULAR ANALYSIS OF 'MUSCLE SYSTEM PROCESS' FOR INTERNAL THORACIC ARTERY AND SAPHENOUS VEIN CONDUITS
}

Sandra Kałużna ${ }^{1}$, Mariusz J. Nawrocki ${ }^{2}$, Karol Jopek ${ }^{1}$, Greg Hutchings ${ }^{2,3}$, Bartłomiej Perek ${ }^{4}$, Marek Jemielity $^{4}$, Bartosz Kempisty ${ }^{1,2,5,6}$, Agnieszka Malińska ${ }^{1,7}$, Paul Mozdziak ${ }^{8}$, Michał Nowicki ${ }^{1}$

\begin{abstract}
Coronary artery bypass graft (CABG) is the surgical method most commonly used to treat coronary artery disease (CAD). The vessels that are used in CABG are usually the internal thoracic artery (ITA) and the saphenous vein (SV). Transplant patency is one of the most important factors affecting transplant success. In this study, we used an expressive microarray method, approved by RT-qPCR, for transcriptome analysis of arterial and venous grafts. In the search for potential molecular factors, we analyzed gene ontologies of different expression based on the muscular system. Among interesting groups, we distinguished muscle cell proliferation, muscle contraction, muscle system process, regulation of smooth muscle cell proliferation and smooth muscle cell proliferation. The highest increase in gene expression was observed in: ACTN2, RBPMS2, NR4A3, KCNA5, while the smallest decrease in expression was shown by the P2RX1, KCNH2, DES and MYOT genes. Particularly noteworthy are the ACTN2 and NR4A3 genes, which can have a significant impact on vascular patency. ACTN2 is a gene that can affect the formation of atherosclerotic plaques, while NR4A3 occurs in 4 of the 5 ontological groups discussed and can affect the inflammatory process in the blood vessel. To summarize, the presented study provided valuable insight into the molecular aspects characterizing the vessels used in CABG, and in particular identified genes that may be the target for further studies on duct patency.
\end{abstract}

Running title: CABG grafts' molecular analysis of 'muscle system process'

Keywords: coronary artery bypass grafting, internal thoracic artery, saphenous vein, microarray

\footnotetext{
${ }^{1}$ Department of Histology and Embryology, Poznań University of Medical Sciences, Poznań, Poland

${ }^{2}$ Department of Anatomy, Poznań University of Medical Sciences, Poznań, Poland

${ }^{3}$ The School of Medicine, Medical Sciences and Nutrition, University of Aberdeen, Aberdeen, UK

${ }^{4}$ Department of Cardiac Surgery and Transplantology, Poznan University of Medical Sciences, Poznań, Poland

${ }^{5}$ Department of Obstetrics and Gynecology, University Hospital and Masaryk University, Brno, Czech Republic

${ }^{6}$ Department of Veterinary Surgery, Institute of Veterinary Medicine, Nicolaus Copernicus University in Toruń, Toruń, Poland

${ }^{7}$ Division of Anatomy and Histology, University of Zielona Góra, Zielona Góra, Poland

${ }^{8}$ Physiology Graduate Program, North Carolina State University, NC, USA

* Correspondence: bkempisty@ump.edu.pl

Full list of author information is available at the end of article
} 


\section{Introduction}

Graft patency is an important aspect to consider when using coronary artery bypass graft (CABG), the surgical method most commonly used to treat coronary artery disease (CAD). The aim of the procedure is to improve blood circulation in atherosclerotic coronary arteries [1,2]. CABG gives better clinical results compared to other methods, e.g. percutaneous coronary intervention (PCI) [3]. This is reflected in the reduction in mortality in some subgroups of patients, and improvement is also noticeable in patients with complex coronary lesions, e.g. in patients with recurrent myocardial infarction or angina pectoris [2]. However, the use of the CABG method is also associated with numerous perioperative and postoperative complications that may prevent the patient from fully recovering [3].

The vessels used in CABG are usually the internal thoracic (mammary) artery (ITA/IMA) and saphenous vein (SV), $[1,2,4,5]$, which usually replace the left anterior descending (LAD) [6]. The choice of vessel may depend on the patient's age, as SV is most often chosen in the elderly while young people undergo ITA transplantation [7]. The saphenous vein is a large, subcutaneous and surface vein of the leg which is transplanted in the reverse position so that its venous valves don't obstruct the flow [5]. For arterial transplantation doctors use left internal mammary artery (LIMA), right internal mammary artery (RIMA) or perform bilateral transplant (BIMA) [8].

Choosing the right vessel for transplantation can be difficult, but the choice is based on the surgeon's skills, the unit in which the surgery will take place, and the patient's individual approach. [9] It is well known that SV is a vessel that is easier to wash and its endoscopic uptake does not affect its patency [10]. However, an advantage for ITA may be the fact that they have their own blood source from a blood vessel, they have a well-built internal elastic plaque, less myocytes in the adventitia [11], which makes them less susceptible to atherosclerosis.

Complications that are observed in patients who received SV (acute thrombosis, intimal hypertrophy, atherosclerosis) may be caused by risk factors, including smoking, increased blood cholesterol, diameter of the vessel and the surgical technique used [3]. In contrast, ITA transplants may be subject to narrowing, especially in response to vasoactive drugs [11].

Scientists and doctors are constantly trying to learn about the molecular, physiological, pathophysiological mechanisms affecting the patency of the transplant [12-14], and to develop innovative surgical methods that can extend the functionality of the transplant $[15,16]$. Sabik et al. using the method longitudinal analysis showed a patency index 1,5 and 10 years after surgery. They compared the use of ITA and SV in a population of 50278 patients. The results for ITA were 93\%, 88\% and 90\% respectively, while for SV 78\%, 65\% and 57\% [17].
The comparison indicates that a better patency index is obtained by using ITA during CABG, however, if ITA arteries are taken from both sides of the body it may result in mediastinitis, which is a life-threatening complication [18]. The pathophysiology of the saphenous vein is becoming better understood over the years, however, the failure of the SV transplant still achieves high results: $35-50 \%$. SV is exposed to arterial circulation, structural changes within the vessel that include an inflammatory response with intimal hyperplasia. To prevent graft remodeling stenosis is successfully used, which reduces hypertrophy and the development of vascular lumen irregularities [15].

The success of the transplant is influenced by many factors, however it is the patency of the transplant that is still a major impediment to the full success of the surgery. This is why it is an important topic of our research, especially in the field of molecular research. Therefore, in our work we tried to determine a marker useful for assessing the patency of the graft based on the muscular system of the blood vessels in question. We focused on 5 ontological groups: "muscle cell proliferation", "muscle contraction", "muscle system process", "regulation of smooth muscle cell proliferation" and "smooth muscle cell proliferation" comparing the internal thoracic artery and saphenous vein conduits.

\section{Materials and methods \\ Operation procedure and sample collection}

In most patients, the left ITA was used to bypass the left anterior descending coronary artery (LAD). The other target coronary arteries were usually revascularized with SV grafts.

All surgeries were performed through median sternotomy. SV grafts were obtained through a fulllength thigh incision over its course. Pivotal points of the procedure included minimal manipulation of the graft ("no-touch" technique), avoiding extensive dilation of the conduits, using low-intensity electrocautery and the control of the branches with stainless-steel vascular clips. In all cases, distal part of the obtained SV segment (at least 15-20 mm in length) was saved for further laboratory studies.

ITA conduits were harvested as pedicled, together with satellite veins and endothoracic fascia from the 2 nd to 6 th intercostal space. The distal end of the ITA segment was divided at the level of its bifurcation. After heparinization, ITA conduits were clipped distally, injected with $10 \mathrm{~mL}$ of a papaverine solution $(1 \mathrm{mg} / \mathrm{mL})$, and allowed to pharmacologically dilate. Immediately before anastomosis of the distal end of ITA to the recipient coronary artery, a 10-mm segment of the conduit was harvested for further molecular and histological tests.

The sets of the vessel samples, both SV and ITA, were immediately snap-frozen in liquid nitrogen and stored at $-80{ }^{\circ} \mathrm{C}$ until RNA isolation. Another 
set of samples was directed for histochemical examination. Transcriptome screening analysis was performed on 18 SV and 20 ITA samples.

\section{Microarray expression study and data analysis}

Our experiment employed 38 GeneChip $\AA$ HGU219 (Affymetrix, Santa Clara, CA, USA) microarrays to simultaneously examine thousands of transcripts for each of the analyzed samples. In the first step, the total RNA (500 ng) from each pooled sample was subjected to two rounds of sense cDNA amplification (Ambion $\AA$ WT Expression Kit, provided by Ambion, Austin, TX, USA). The synthesis of cRNA was performed by in vitro transcription $(16 \mathrm{~h}, 40$ $\left.{ }^{\circ} \mathrm{C}\right)$. Then, cRNA was purified and re-transcribed
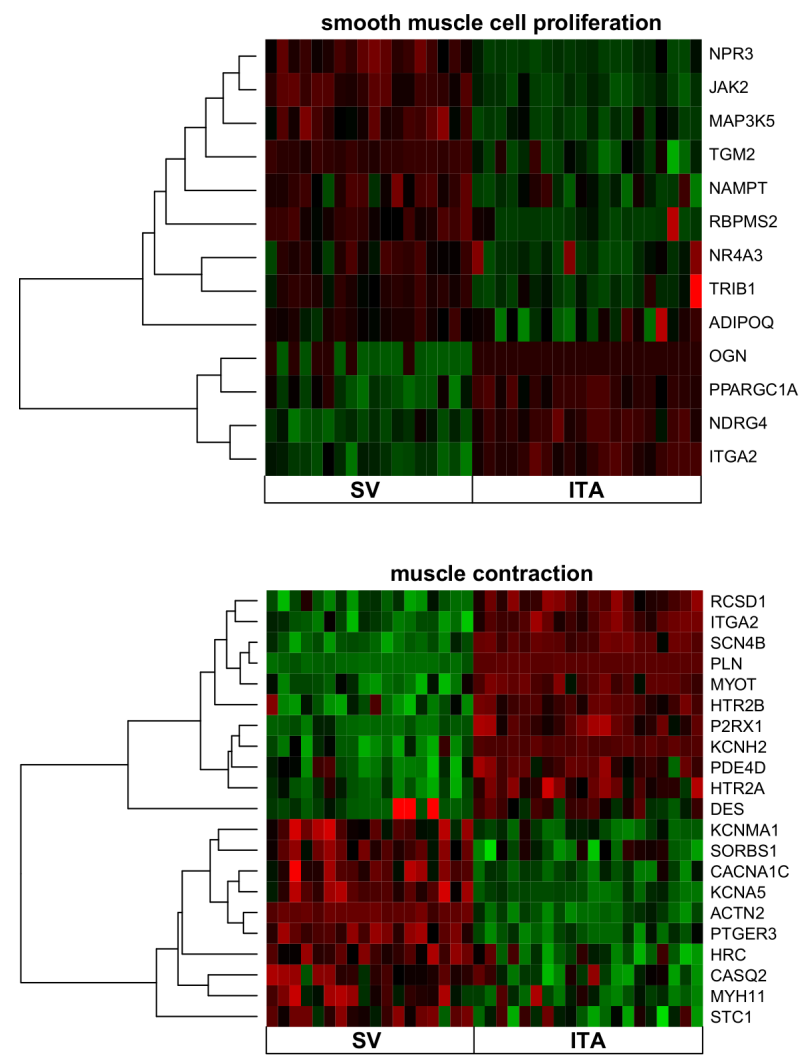

into cDNA. Subsequently, cDNA samples were used for biotin labeling and fragmentation using an Affymetrix GeneChip® WT Terminal Labeling and Hybridization kit (Affymetrix). Next, the biotin-labeled samples were loaded onto and hybridized to the Affymetrix ${ }^{\circledR}$ Human Genome U219 Array Strip. Hybridization was conducted at $48^{\circ} \mathrm{C}$ for $20 \mathrm{~h}$, employing an AccuBlock ${ }^{\mathrm{TM}}$ Digital Dry Bath (Labnet International, Inc., Edison, NJ, USA) hybridization oven. Then, microarrays were washed and stained, according to technical protocol, using an Affymetrix GeneAtlas ${ }^{\mathrm{TM}}$ Fluidics Station (Affymetrix, Santa Clara, CA, USA). The strips were scanned using an Affymetrix GeneAtlas ${ }^{\mathrm{TM}}$ Imaging Station (Affymetrix, Santa Clara, CA, USA). The scans of the microarrays
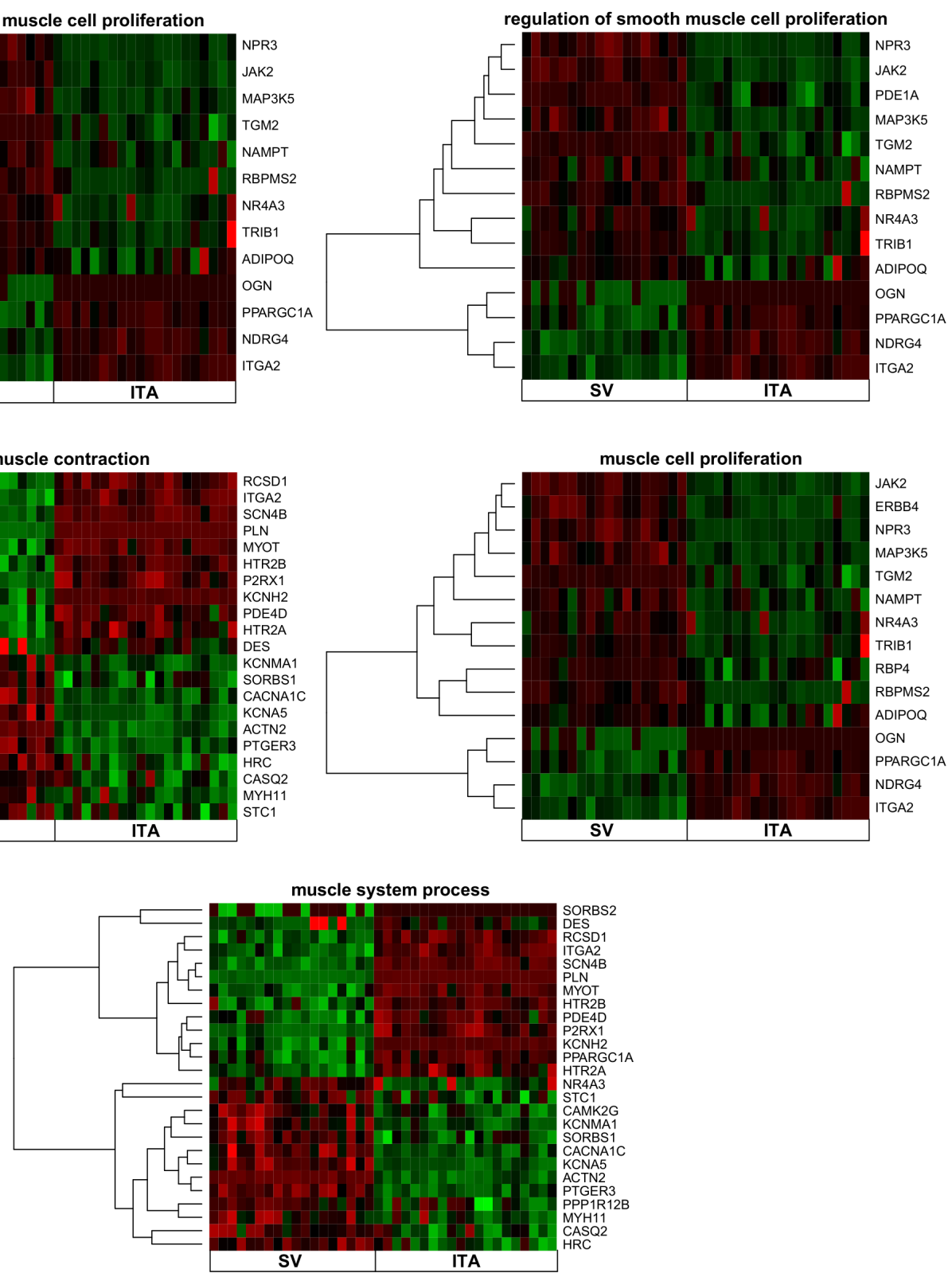

FIGURE 1 Heatmaps presenting differentially expressed genes involved in "muscle cell proliferation", "muscle contraction", "muscle system process", "regulation of smooth muscle cell proliferation" and "smooth muscle cell proliferation" based on GO BP terms. Each row on the Y axis represents a single transcript. The red color indicates downregulated genes while the green are upregulated 


\begin{tabular}{|c|c|c|c|c|}
\hline Gene symbol & Gene name & Fold change & Adj. p.val \\
\hline ACTN2 & actinin, alpha 2 & 10.99 & $<0.05$ \\
\hline RBPMS2 & RNA binding protein with multiple splicing 2 & 6.57 & $<0.05$ \\
\hline NR4A3 & nuclear receptor subfamily 4, group A, member 3 & $<.72$ & $<0.05$ \\
\hline KCNA5 & potassium voltage-gated channel, shaker-related subfamily, member 5 & 5.46 & $<0.05$ \\
\hline NPR3 & natriuretic peptide receptor C/guanylate cyclase C (atrionatriuretic peptide receptor C) & 5.30 & $<0.05$ \\
\hline PTGER3 & prostaglandin E receptor 3 (subtype EP3) & 3.53 & $<0.05$ \\
\hline RBP4 & retinol binding protein 4, plasma & $<.23$ & $<0.05$ \\
\hline OGN & osteoglycin & 3.23 & $<0.05$ \\
\hline JAK2 & Janus kinase 2 & 3.18 & $<0.05$ \\
\hline MYH11 & myosin, heavy chain 11, smooth muscle & $<0.05$ \\
\hline PPARGC1A & peroxisome proliferator-activated receptor gamma, coactivator 1 alpha & -2.20 & $<0.05$ \\
\hline NDRG4 & NDRG family member 4 & -2.22 & $<0.05$ \\
\hline HTR2A & 5-hydroxytryptamine (serotonin) receptor 2A, G protein-coupled & -2.32 & $<0.05$ \\
\hline ITGA2 & integrin, alpha 2 (CD49B, alpha 2 subunit of VLA-2 receptor) & -2.38 & $<0.05$ \\
\hline HTR2B & 5-hydroxytryptamine (serotonin) receptor 2B, G protein-coupled & -2.64 & $<0.05$ \\
\hline SCN4B & sodium channel, voltage-gated, type IV, beta subunit & -3.13 & $<0.05$ \\
\hline MYOT & myotilin & -3.39 & $<0.05$ \\
\hline DES & potassium voltage-gated channel, subfamily H (eag-related), member 2 & -6.25 & $<0.05$ \\
\hline KCNH2 & purinergic receptor P2X, ligand-gated ion channel, 1 & -6.48 & $<0.05$ \\
\hline P2RX1 & ind & -4.44 & $<0.05$ \\
\hline
\end{tabular}

TABLE 1 The 10 most significantly upregulated and 10 most significantly downregulated genes involved in muscle system process
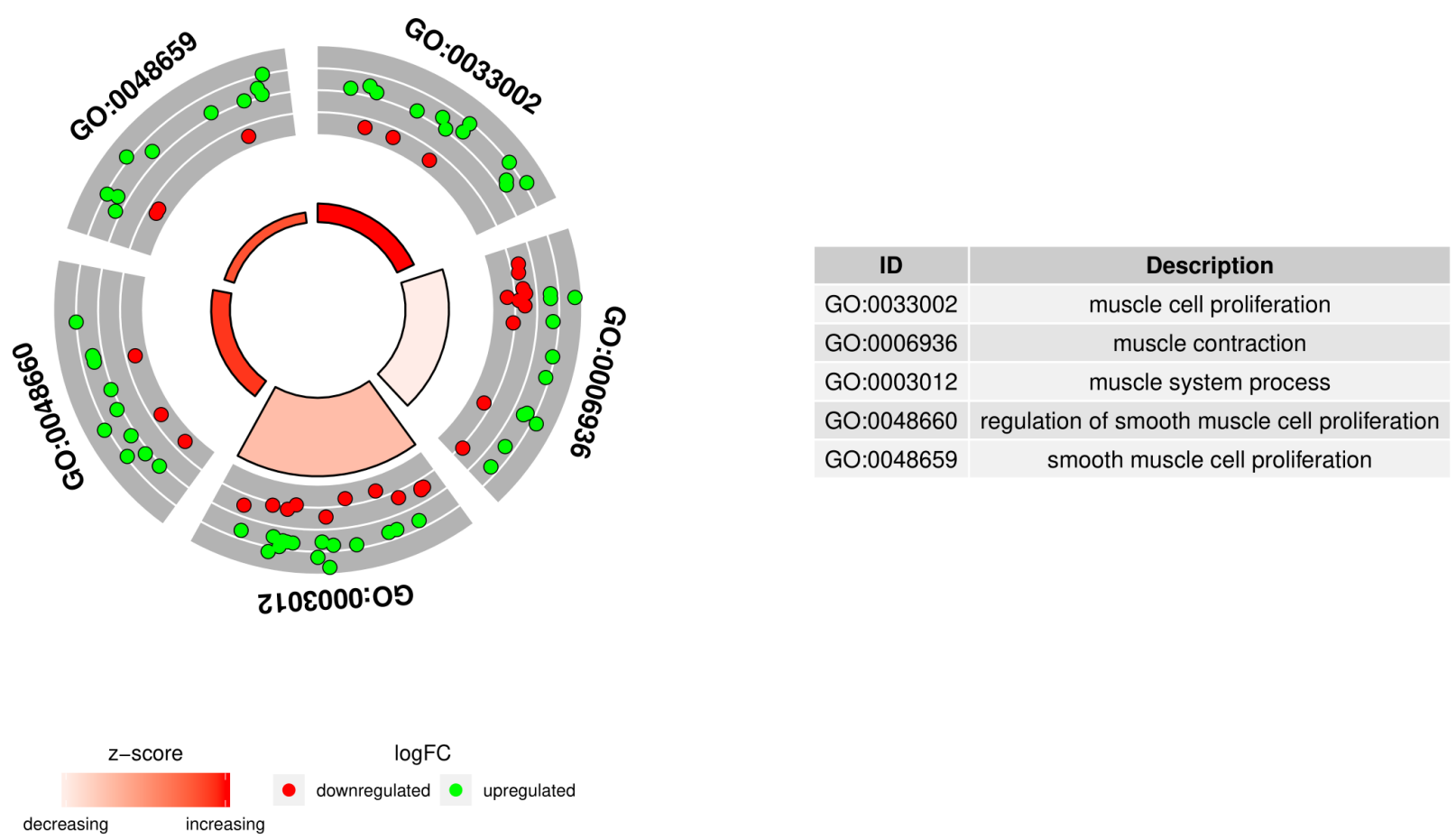

FIGURE 2 The circular scatter plots of differentially expressed genes involved in "muscle cell proliferation", "muscle contraction", "muscle system process", "regulation of smooth muscle cell proliferation" and "smooth muscle cell proliferation" GO BP terms. Each dot represents a single gene. The z-scores were presented as segments of inner circles

were saved on hard drives as *.CEL files for downstream data analysis.

Quality control (QC) studies were performed using the Affymetrix GeneAtlas ${ }^{\mathrm{TM}}$ Instrument Control
Software 2.0.0.460 (Affymetrix, Santa Clara, CA, USA), according to the manufacturer's standards. The generated *.CEL files were subjected to further analysis performed using the R statistical lan- 
guage and Bioconductor package with the relevant Bioconductor libraries. To correct the background, normalize, and summarize the results, we used the robust multiarray averaging (RMA) algorithm. Assigned biological annotations were obtained from the "pd.ragene.2.1.st" library and employed for the mapping of normalized gene expression values with their symbols, gene names, and Entrez IDs, allowing to generate a complex gene data table. To determine the statistical significance of the analyzed genes, moderated t-statistics from the empirical Bayes method were performed. The obtained p-values were corrected for multiple comparisons using Benjamini and Hochberg's false discovery rate and described as adjusted p-values. The selection of significantly altered genes was based on a p-value beneath 0.05 and an expression higher than two-fold. The differentially expressed gene list (separated for upregulated and downregulated genes) was uploaded to the DAVID Bioinformatics Resources 6.8 software (Database for Annotation, Visualization and Integrated Discovery) [19], where the significantly upregulated Gene Ontology (GO) terms were extracted. The selection of significantly altered GO terms was based on a p-value (Benajamini) $<0.05$ and the volume of at least five genes.

To further investigate the chosen gene sets, we investigated their mutual relations with the GOplot package [20]. Subsequently, sets of differentially expressed genes from selected GO BP terms were applied to the STRING10 software (Search Tool for the Retrieval of Interacting Genes/Proteins) for interactions prediction. STRING is a huge database containing information on protein/gene interactions, including experimental data, computational prediction methods, and public text collections.
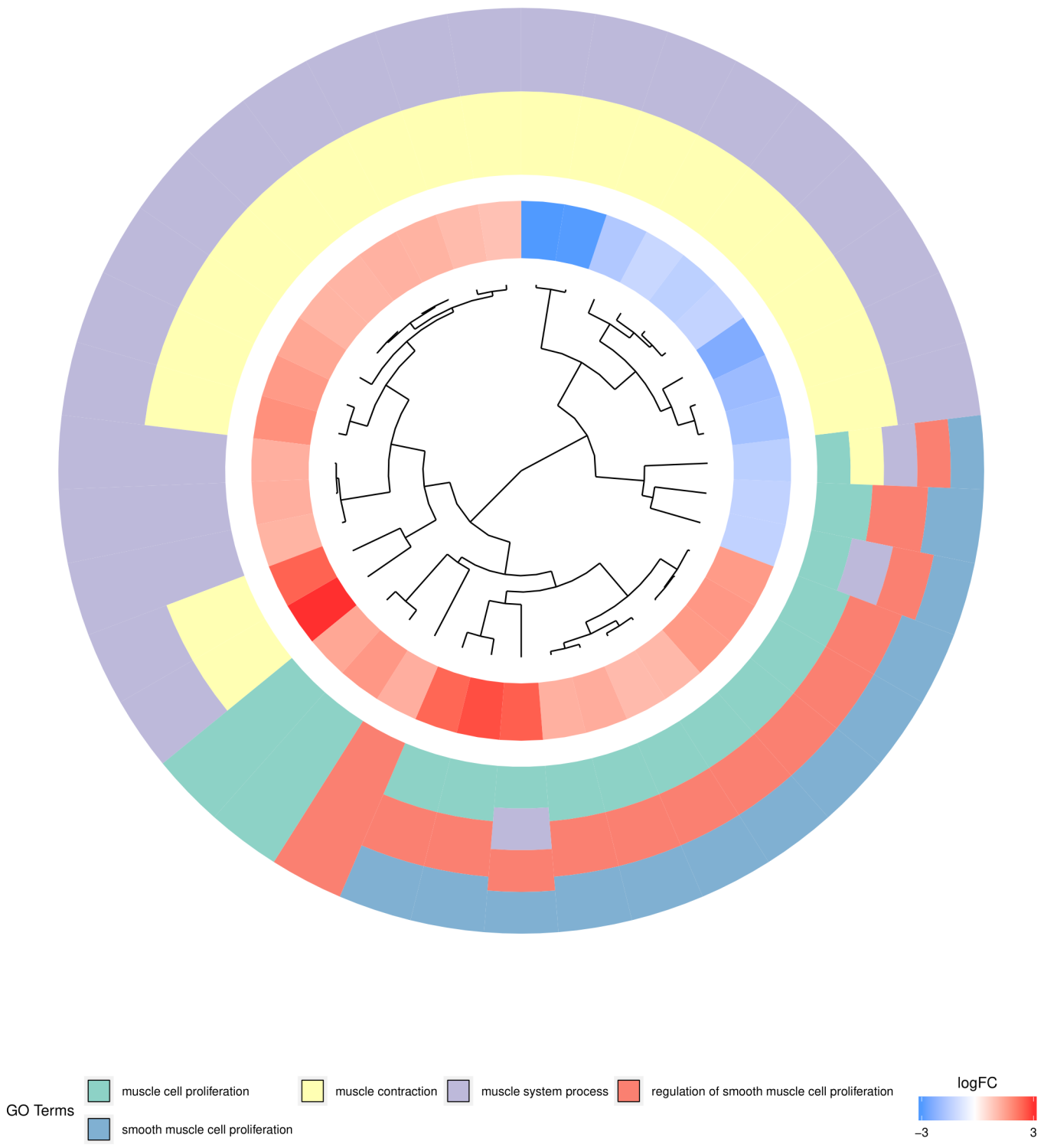

FIGURE 3 The dendrogram of differentially expressed genes involved in "muscle cell proliferation", "muscle contraction", "muscle system process", "regulation of smooth muscle cell proliferation" and "smooth muscle cell proliferation" GO BP terms. The DEGs were clustered based on their $\operatorname{logFC}$ values 


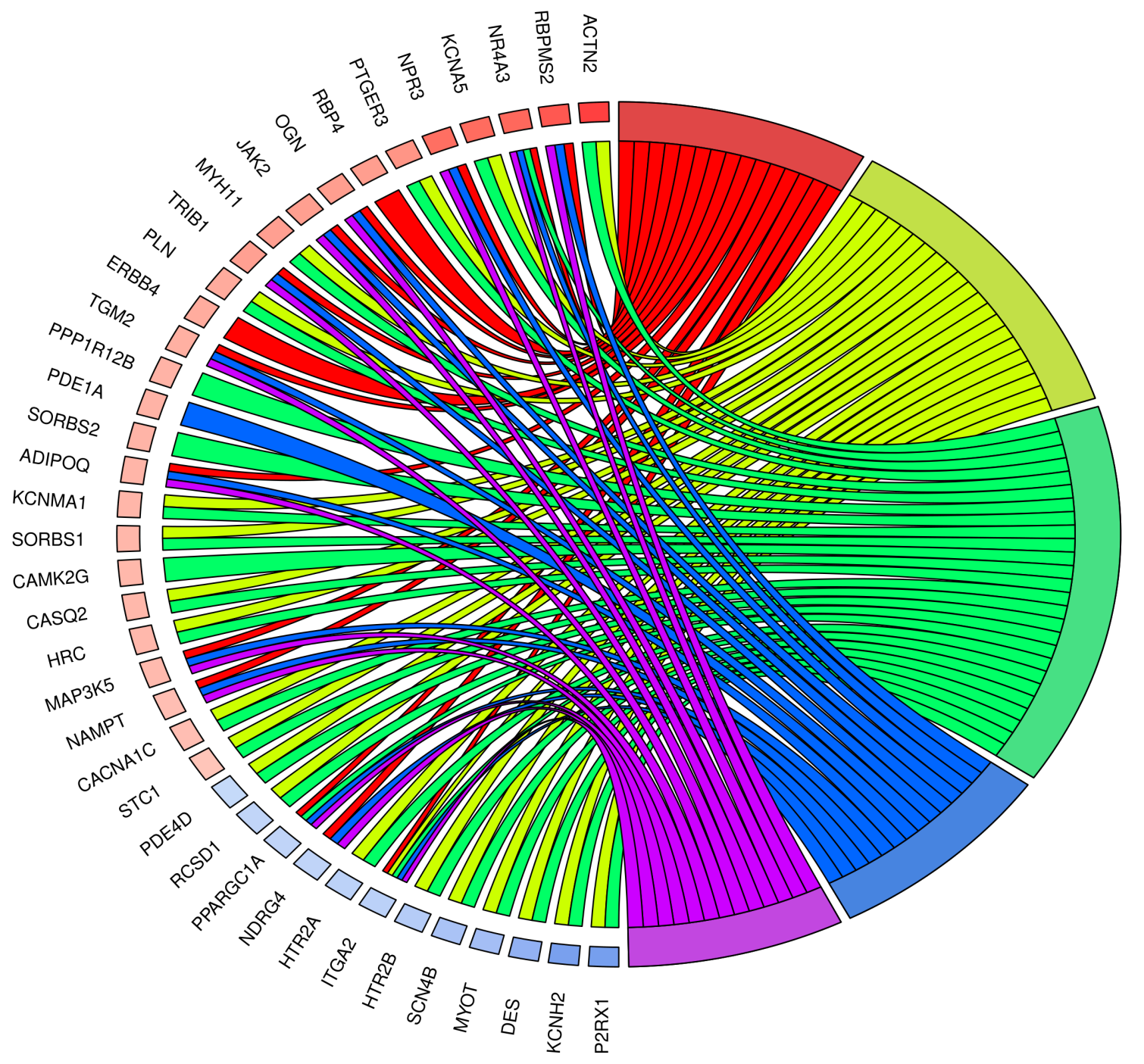

GO Terms

muscle cell proliferation $\quad \square$ muscle contraction

smooth muscle cell proliferation

regulation of smooth muscle cell proliferation

$\log \mathrm{FC}$

FIGURE 4 Analysis of enriched gene ontological groups involved in muscle system process. The network plot presenting the linkages of genes and GO BP terms

\section{Ethical approval}

The research related to human use has been complied with all the relevant national regulations, institutional policies and in accordance the tenets of the Helsinki Declaration, and has been approved by the authors' institutional review board or equivalent committee. Bioethical Committee approval no. $1201 / 08$, approved on $18 / 12 / 2008$.

\section{Results}

We used Human Genome U219 Array Strip for the microarray gene expression analysis of internal thoracic artery (ITA) and the saphenous vein (SV) conduits. This method allowed us to study the gene expression of 49,308 transcripts. We selected genes with more than 2-fold changes and correc- ted p-values less than 0.05 for downstream analysis. A total of 1170 differentially expressed genes (DEGs) were identified according to the above criteria. We started the microarray gene expression analysis with subjecting the list of DEGs to DAVID software, which showed that the genes can be assigned to many gene ontology groups (GO BP terms). This paper focused on the genes involved in muscle system process. The DAVID software indicated the following GO BP terms, which cover the above processes: "muscle cell proliferation", "muscle contraction", "muscle system process", "regulation of smooth muscle cell proliferation" and "smooth muscle cell proliferation". The 39 genes involved in those processes were clustered using hierarchical clustering and presented as heatmaps (Fig. 1). 


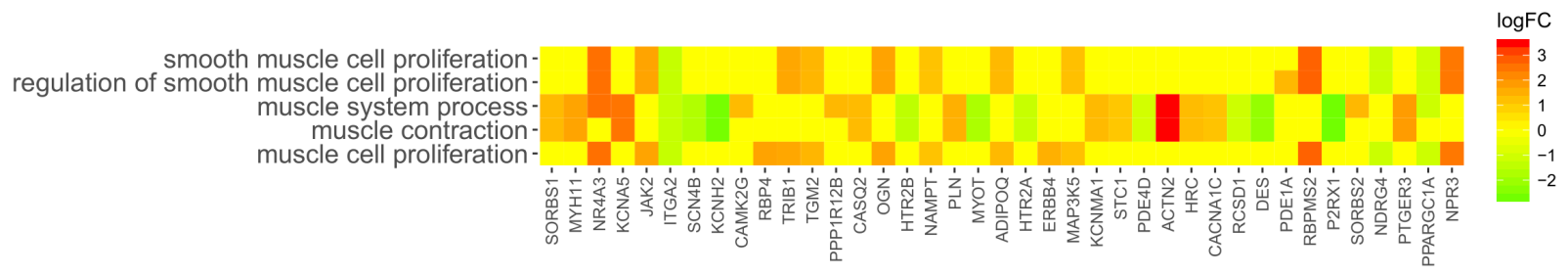

FIGURE 5 Heatmap presenting the relationship between genes and selected GO BP terms. The yellow color of tiles indicates the absence of $\operatorname{logFC}$ values

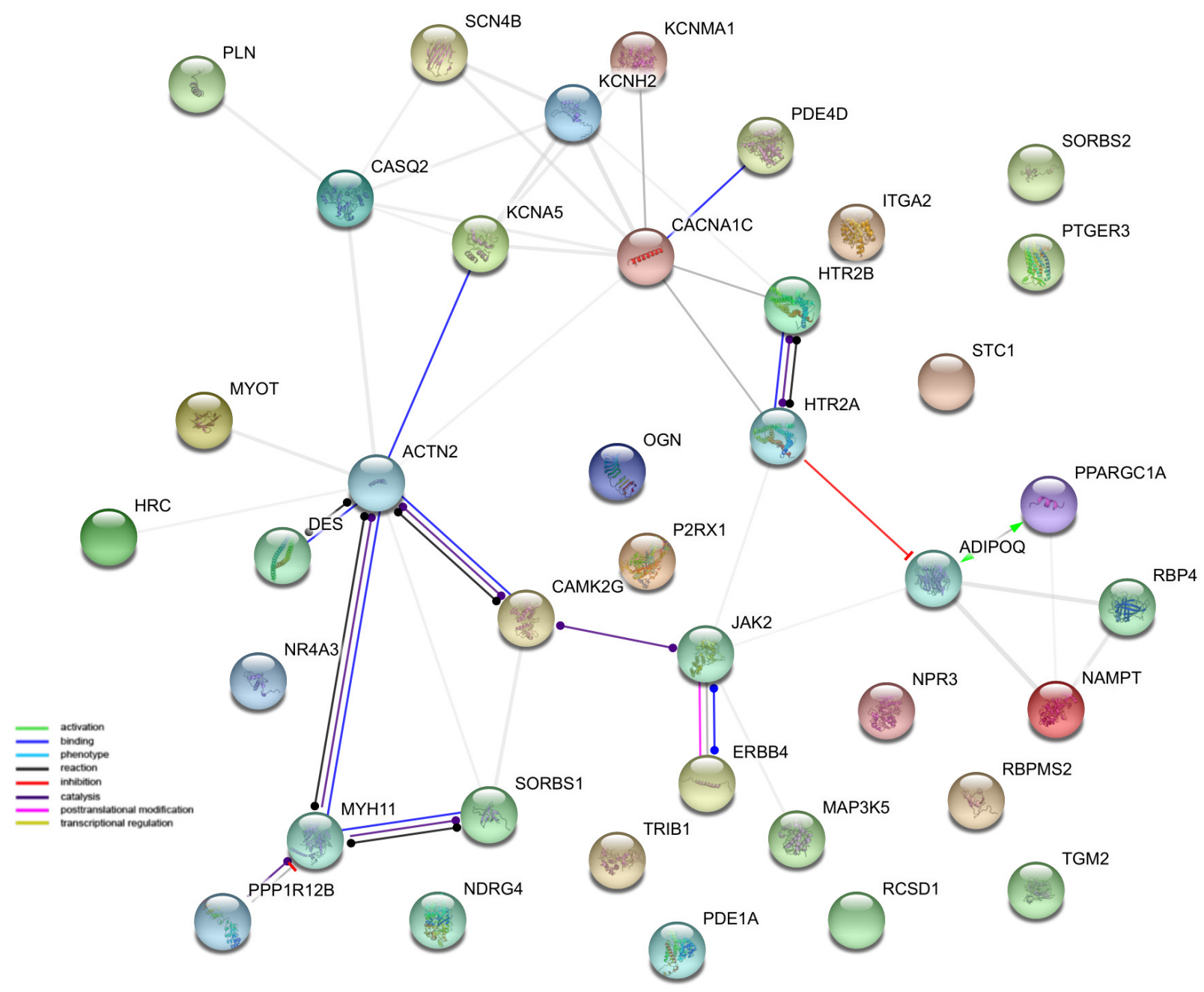

FIGURE 6 Interaction network of proteins encoded by DEGs belonging to "muscle cell proliferation", "muscle contraction", "muscle system process", "regulation of smooth muscle cell proliferation" and "smooth muscle cell proliferation" GO BP terms. The network was generated by STRING software. Network nodes represent proteins, while empty nodes indicate proteins of unknown 3D structure

It is worth mentioning that 12 genes were downregulated while 27 genes were upregulated. The 10 most significantly upregulated and downregulated genes, their symbols, fold changes and corrected pvalues are shown in table 1.

In the next part of the analysis, we focused on the z-scores, which tell us whether the biological process is more likely to be decreased (negative value) or increased (positive value). The z-scores were presented as segments of inner circles in the figure 2 . The z-scores of above-mentioned GO BP terms had positive values, so the processes described by these GO BP terms were upregulated.

In the next section, we checked the interaction between selected ontological groups. One of the most visually appealing ways of presenting such interaction is dendrogram (Fig. 3). Clusters contain functionally related genes based on their expression pattern. The middle circle represents a logarithm of fold change ( $\log \mathrm{FC})$ of differentially expressed genes assigned to 


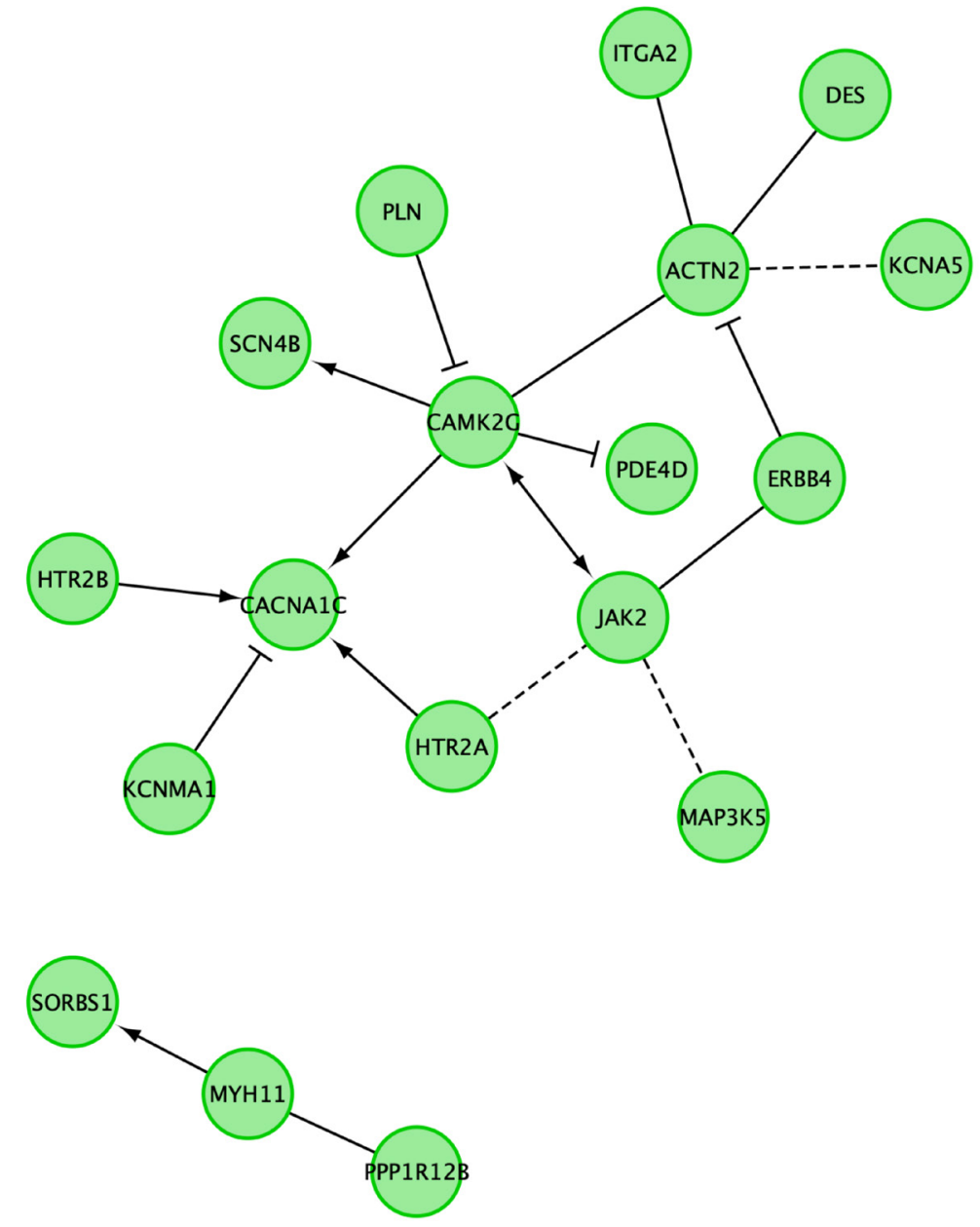

FIGURE 7 Reactome FI networks between differentially expressed genes belonging to the "muscle cell proliferation", "muscle contraction", "muscle system process", "regulation of smooth muscle cell proliferation" and "smooth muscle cell proliferation" GO BP terms. "--->" indicates activating/catalyzing, "-|" for inhibition, "-“ FIs extracted from complexes or inputs and "---" predicted FIs

the studied GO terms. The GO terms are shown as the outer ring. The genes whose expression is downregulated form clusters marked by blue part of the middle circle and analogously, red indicates upregulated genes. Clusters of the same color over the entire width of the outer circle represent genes that are unique for a specific GO term. Clusters of different colors on the cross section of outer circle show sets of genes which are likely to be functionally related. The dendrogram showed that many genes belong simultaneously to "muscle contraction" and "muscle system process". The same applies to "muscle cell proliferation", "regulation of smooth muscle cell proliferation" and "smooth muscle cell proliferation".

In the gene ontology database, single genes may belong to many ontological terms. For this reason, we used plots with visualization of $\log F C$ values and relationship between genes and selected GO BP terms (Fig. 4). The relationship was also presented as a heatmap (Fig. 5). The strongest upregulated genes from examined GO BP terms included, among others: ACTN2- actinin, alpha 2; RBPMS2- RNA binding protein with multiple splicing 2 and NR4A3- nuclear re- ceptor subfamily 4 , group A, member 3 . The most downregulated genes are: $P 2 R X 1$ - purinergic receptor $\mathrm{P} 2 \mathrm{X}$, ligand-gated ion channel, $1 ; \mathrm{KCNH} 2$ - potassium voltage-gated channel, subfamily $\mathrm{H}$ (eag-related), member 2 and DES- desmin.

In the next part of analysis, we focused on the interaction between proteins encoded by DEGs belonging to studied GO BP terms. Firstly, we used STRING software for the interaction prediction (Fig. 6).

In the next part of analysis, we used ReactomeFIViz app for investigation of functional interactions between proteins encoded by DEGs belonging to selected GO BP terms (Fig. 7).

Finally, we mapped gene expression data (ratio more than 1.5) on "calcium signaling pathway" onto pathway graph based on KEGG (Kyoto Encyclopedia of Genes and Genomes) database (Fig. 8).

\section{Discussion}

Despite many studies, there is still little understanding of the molecular causes that affect the patency of the blood vessel used during CABG. The arterial and venous vessels differ primarily in the 


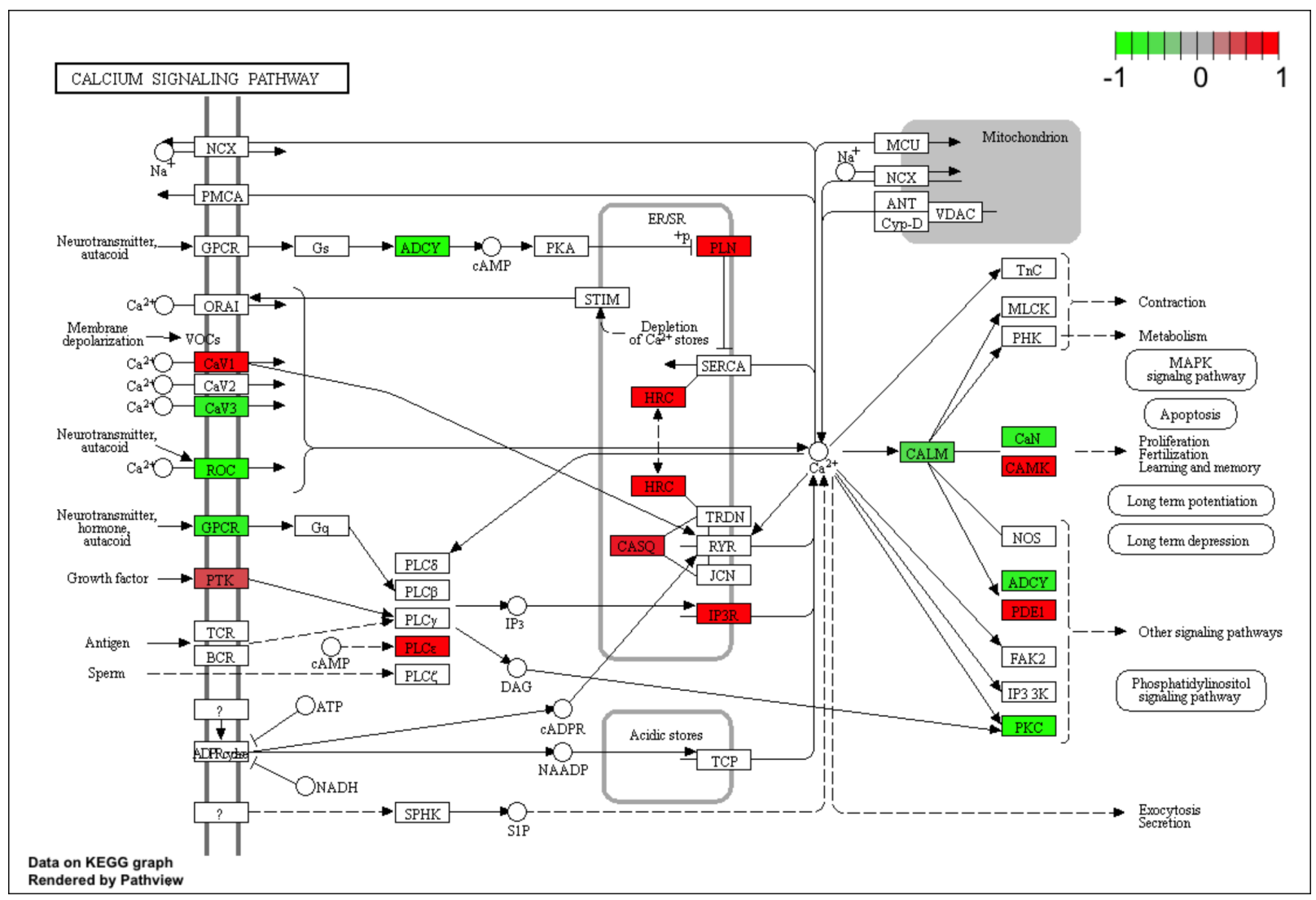

FIGURE 8 Pathway graph of "calcium signaling pathway" based on KEGG database. The red color indicates parts of the pathway that are upregulated in internal thoracic artery conduit while the green color represents components upregulated in saphenous vein conduit

thickness of the middle muscle membrane, which is much more developed in the case of arteries. A comparison of the ITA and SV muscle profiles used during CABG may indicate potential markers of both blood vessels that can have a significant impact on the quality of the ducts and their usefulness in surgical intervention. By analyzing high-throughput molecular arrays, we have obtained 39 genes that have been interpreted based on ontological groups associated with the muscular system: "muscle cell proliferation", "muscle contraction", "muscle system process", "regulation of smooth muscle cell proliferation" and "Smooth muscle cell proliferation". We have obtained 27 genes with increased expression and 10 genes with reduced expression.

The highest rate of increase in gene expression was recorded for the alpha actinin 2 (ACTN2) gene, which was identified in two ontological groups: muscle concentration and muscle system process. ACTN2 together with 3 other alpha actinin genes (ACTN1, ACTN3 and ACTN4) is a member of the spectrin gene superfamily. ACTN2 is a sarcometric isoform, its expression occurs in the myocardium and in skeletal muscle is co-expressed in ACTN3 [21]. The ACTN2 protein is responsible for the anchoring and crosslinking of actin fibers in the heart band at the sarcomer boundaries, and also interacts with the ion channels of the heart [22]. In addition, ACTN2, by in- teracting with other proteins plays an important role in the organization of thin fibers and interactions between the sarcomere and the muscle membrane [23]. By sequencing the entire genome of patients in one family with familial hypertrophic cardiomyopathy, mutations Ala119Thr in this gene were detected in patients with clinical symptoms [22]. Chiu et al. on the other hand, reported 3 more mutations: Thr495Met, Glu583Ala and Glu628Gly in patients from other 4 families [23]. Mutations in this gene may also lead to DCM, idiopathic ventricular fibrillation, juvenile atrial arrhythmias, restrictive cardiomyopathy, and left ventricular failure and sudden unexplained death [24]. Currently, there are numerous new reports on the discovery of mutations in the ACTN2 gene associated with cardiac diseases [24], as well as their impact on the phenotype of a given disease [25]. The formation of atherosclerotic plaques in blood vessels is one of the factors affecting the patency of the vessel. Liu et al. based on cluster analysis, Gene Ontology (GO) and pathway enrichment analysis suggest that deregulation of the immune system and cytoskeleton of smooth muscle cells accelerates the progression of carotid atherosclerotic plaques [26]. This is an interesting reference to our research and may be another alternative to conducting experiments.

Another gene with a high expression increase is RNA binding protein with multiple splicing $2(R B-$ 
PSM2), which unlike ACTN2, was found in 3 other ontological groups: muscle cell proliferation, regulation of smooth muscle cell proliferation and smooth muscle cell proliferation. RBPSM2 belongs to the RRM family and is expressed in the vertebrate heart and gastrointestinal tract [27]. As a class of RBP (RNA-binding proteins), they can affect all stages of the RNA life cycle, including synthesis, translocation, translation, processing, and degradation. In contrast, the mutations present in their sequence may be the cause of cardiovascular disorders. Akerberg et al. state that in zebrafish, encoded proteins may be responsible for regulating aspects related to the differentiation, survival proliferation and contractility of cardiomyocytes [28]. Despite the fact that scientists are trying to explain the role of DNA binding proteins in transcriptome regulation during phenotype change in health and cardiovascular disease [29], there are still few sources explaining the role of RBPSM2 in connection with $\mathrm{CABG}$ and especially in relation to the patency of the blood vessels used.

Another gene worthy of attention is NR4A3 belonging to the nuclear receptor superfamily. NR4A includes three members: Nur77 (also called NR4A1, TR3, NGFI-B, NAK-1), Nurr1 (NR4A2, NOT) and NOR-1 (NR4A3, MINOR) and are composed of a central DNA binding domain, a C-terminal domain ligand binding and a N-terminal transactivation domain [30]. Nur77, Nurr1 and NOR-1 are induced in human smooth muscle cells (SMCs) after activation by inflammatory cytokines and growth factors, and NR4A nuclear receptors are expressed in human vascular and atherosclerotic lesions [30]. Martinez-Gonzalez et al. demonstrated that NOR-1 is transiently expressed in coronary SMCs in response to balloon dilatation [31], which may be of great importance in CABG. NR4A1 and NR4A3 have been described as receptors regulating cell differentiation, proliferation, inflammation and apoptosis, as well as being particularly important for their impact on the development of cardiovascular diseases [32]. It is known that Neuron-derived orphan receptor 1 (NOR1) regulates the proliferation of blood vessel smooth muscle cells (VSMC). One of the latest studies was based on the protective effect of exendin-4 (Ex-4) on mouse vessels, and by inhibiting NOR1 expression in VSMC. The conclusion drawn from the study suggests that Ex-4 suppression of neointima formation following vascular damage and VSMC proliferation is likely due to inhibition of NOR1 expression [33]. In turn, another research team showed that VTN, a gene coding for the well-known multifunctional glycoprotein (vimentin) of key importance for cell adhesion, migration and proliferation, but also involved in thrombosis and fibrinolysis in humans can participate in NOR-1 mediated vascular responses [34]. Studies of this syndrome have previously also shown the role of NOR-1 as a negative modulator of acute response induced by pro-inflammatory stimuli in the vascular system [35]. In our study NR4A3 identified in 4 of 5 ontological groups (muscle cell proliferation, muscle contraction, regulation of smooth muscle cell proliferation and smooth muscle cell proliferation) which indicates a broad spectrum of the gene's impact on the muscular system of blood vessels.

We also noticed the KCNA5 and KCNH2 genes belonging to the same ontological groups: muscle contraction and muscle system process. The first observed the fourth largest increase in expression of this gene, while in the case of $\mathrm{KCNH} 2$ the second largest decrease in gene expression was noted. These genes encode voltage gated potassium channels (VGPC) Kv1.5 for KCNA5 and Kv11.1 for $K C N H 2$, whose mutations may affect the occurrence of severe arrhythmias [36]. Voltage gated channels are a frequent aspect of research in relation to vascular diseases, e.g. as DHEA affects the expression of Kv1.5 channels and how it relates to remodeling of blood vessels, especially SV and cervical vessels [37]. Further studies also based on the effect on Kv1.5 led to the conclusion that said channel is sensitive to DP0-1, which plays an important role in determining the microvascular tension and response to vasoconstrictive and vasodilating agents [38]. In turn, studies on porcine coronary vessels suggest the dominant role of KV1 channels in modulating coronary output voltage and possibly vasodilatation in response to increased metabolism and transient ischemia [39]. Unlike KCNA5, the KCNH2 encoded channels (ERG channels) were examined to determine expression and their action in mouse arteries. In this case, it was concluded that ERG channels may be non-functional in contractile smooth muscles, however, they give a proliferative story [40].

Genes with the largest decrease in gene expression belong to two ontological groups: muscle contraction and muscle system process, and the largest decrease was recorded for purinergic $\mathrm{P} 2 \mathrm{X}$ receptor (P2RX1). P2RX1 belongs to the family of P2X receptors ligated through the P2X channel (7 subtypes) that mediate the action of the purine nucleotide (ATP) as well as its metabolites. It is known that smooth muscle cells in various rat blood vessels express P2RX, including P2RX1 [41] P2X receptors (P2RX) are ion channels that increase intracellular $\mathrm{Ca} 2+$ concentrations by direct gating to extracellular $\mathrm{Ca} 2+[42]$. The $\mathrm{P} 2 \mathrm{X}$ receptor is of interest to researchers in relation to blood vessel smooth muscle cells (VSMC) e.g. synthetic ATP analogues and P2X receptor agonists (Bz-ATP, $\alpha, \beta$-meATP and $\beta$, $\gamma$-meATP) have been shown to inhibit VSMC calcification in vitro [43]. However, there is little information regarding the effect of P2RX1 on the patency of the blood vessel, so this requires further study.

Also noteworthy is the DES gene with a third decrease in gene expression and MYOT with a fourth 
decrease in gene expression. The DES gene codes for desmine, which belongs to the family of intermediate fibers, found in smooth, skeletal and cardiac muscle tissue. Mutations occurring in DES in humans lead to autosomal cardiomyopathy, which results in heart failure, arrhythmia and aortic valve blockage. The MYOT gene coding for motilin protein, whose mutation is one of the causes of myofibrillary myopathy (MFM) [44].

\section{Conclusions}

In the study presented in this article, we performed a transcriptomic analysis of two major blood vessels: the internal thoracic artery and the saphenous vein used for transplantation in CABG, looking for potential molecular factors that may be useful in assessing the patency of the blood vessel. We focused on the molecular analysis of the muscular system process based on the ontological groups associated with this process: muscle cell proliferation, muscle contraction, muscle system process, regulation of smooth muscle cell proliferation and smooth muscle cell proliferation. We distinguished several genes with different expression in both vessels, described above, of which the ACTN2 and NR4A3 gene deserve special attention. ACTN2 is a gene that can affect the formation of atherosclerotic plaques, while $N R 4 A 3$ occurs in 4 of the 5 ontological groups discussed and can affect the inflammatory process in the blood vessel. Both genes can have a significant impact on vascular patency but require further confirmatory testing.

\section{Acknowledgements}

This publication is part of the project: (1) "WroVasc-Integrated Cardiovascular Centre", co-financed by the European Regional Development Fund, within Innovative Economy Operational Program, 2007-2013 realized in Regional Specialized Hospital, Research and Development Center in Wroclaw.

\section{Corresponding author}

Bartosz Kempisty PhD, Department of Histology and Embryology, Department of Anatomy, Poznań University of Medical Sciences, 6 Święcickiego St., 60-781 Poznań, Poland Tel./Fax: +48 61 8546418 / +48 61 8546440, e-mail: bkempisty@ump.edu.pl.

\section{Conflict of interest statement}

The authors declare they have no conflict of interest.

\section{References}

1. Malinska A, Podemska Z, Perek B, Jemielity M, Buczkowski P, Grzymislawska M, Sujka-Kordowska P, Nowicki M. Preoperative factors predicting saphenous vein graft occlusion in coronary artery bypass grafting: a multivariate analysis. Histochem Cell Biol. 2017;148:417-24; DOI:10.1007/s00418-017-1574-4.

2. Kappetein AP, van Mieghem NM, Head SJ. Revascularization Options. Heart Fail Clin. 2016;12:135-9; DOI:10.1016/j.hfc.2015.08.011.

3. Margaritis M, Channon KM, Antoniades C. Statins and vein graft failure in coronary bypass surgery. Curr Opin Pharmacol. 2012;12:172-80; DOI:10.1016/j.coph.2012.01.009.

4. Davierwala PM, Mohr FW. Bilateral internal mammary artery grafting Rationale and evidence. Int J Surg. 2015;16:133-9; DOI:10.1016/J. IJSU.2015.01.012

5. Al-Sabti HA, Al Kindi A, Al-Rasadi K, Banerjee Y, Al-Hashmi K, Al-Hinai A Saphenous vein graft vs. radial artery graft searching for the best second coronary artery bypass graft. J Saudi Hear Assoc. 2013;25:247-54; D0I:10.1016/j.jsha.2013.06.001.

6. Goldman S, Zadina K, Moritz T, Ovitt T, Sethi G, Copeland JG, Thottapurathu L, Krasnicka B, Ellis N, Anderson RJ, Henderson W, VA Cooperative
Study Group \#207/297/364. Long-term patency of saphenous vein and left internal mammary artery grafts after coronary artery bypass surgery: results from a Department of Veterans Affairs Cooperative Study. J Am Coll Cardiol. 2004;44:2149-56; DOI:10.1016/j.jacc.2004.08.064.

7. Nawrocki MJ, Perek B, Sujka-Kordowska P, Konwerska A, Kałużna S Zawierucha P, Bruska M, Zabel M, Jemielity M, Nowicki M, Kempisty B, Malińska A. Differences in expression of genes involved in bone development and morphogenesis in the walls of internal thoracic artery and saphenous vein conduits may provide markers useful for evaluation graft patency. Int J Mol Sci. 2019;20; DOI:10.3390/ijms20194890.

8. Martínez-González B, Reyes-Hernández CG, Quiroga-Garza A, Rodríguez-Rodríguez VE, Esparza-Hernández CN, Elizondo-Omaña RE, Guzmán-López S. Conduits Used in Coronary Artery Bypass Grafting: A Review of Morphological Studies. Ann Thorac Cardiovasc Surg. 2017;23:55-65; DOI:10.5761/atcs.ra.16-00178.

9. Malinska A, Perek B, Buczkowski P, Kowalska K, Ostalska-Nowicka D, Witkiewicz W, Nowicki M. CD68 expression in aortocoronary saphenous vein bypass grafts. Histochem Cell Biol. 2013;140:183-8; DOI:10.1007/ s00418-012-1069-2.

10. Khan SZ, Rivero M, McCraith B, Harris LM, Dryjski ML, Dosluoglu HH. Endoscopic vein harvest does not negatively affect patency of great saphenous vein lower extremity bypass. J Vasc Surg. 2016;63:1546-54 DOI:10.1016/j.jvs.2016.01.032.

11. Bawany FI, Khan MS, Khan A, Kazi AN, Naeem M. Using skeletonised grafts for coronary artery bypass grafting. J Pak Med Assoc. 2014;64:606-10.

12. Tu Q-M, Wang Z-W. Study on mechanism of $\mathrm{c}-\mathrm{Myc}$ in restenosis after coronary artery bypass grafting. Eur Rev Med Pharmacol Sci. 2016;20:2363-7.

13. Sanders WG, Morisseau C, Hammock BD, Cheung AK, Terry CM. Soluble epoxide hydrolase expression in a porcine model of arteriovenous graft stenosis and anti-inflammatory effects of a soluble epoxide hydrolase inhibitor. Am J Physiol Physiol. 2012;303:C278-90; DOI:10.1152/ ajpcell.00386.2011.

14. Samano N, Geijer H, Liden M, Fremes S, Bodin L, Souza D. The no-touch saphenous vein for coronary artery bypass grafting maintains a patency, after 16 years, comparable to the left internal thoracic artery: A randomized trial. J Thorac Cardiovasc Surg. 2015;150:880-8; DOI:10.1016/j. jtcvs.2015.07.027.

15. Taggart DP, Webb CM, Desouza A, Yadav R, Channon KM, De Robertis F, Di Mario C. Long-term performance of an external stent for saphenous vein grafts: the VEST IV trial. J Cardiothorac Surg. 2018;13:117; DOI:10.1186/s13019-018-0803-9.

16. Tinica G, Chistol R, Bulgaru Iliescu D, Furnica C. Long-term graft patency after coronary artery bypass grafting: Effects of surgical technique. Exp Ther Med. 2018;17:359-67; DOI:10.3892/etm.2018.6929.

17. Sabik JF, Lytle BW, Blackstone EH, Houghtaling PL, Cosgrove DM. Comparison of saphenous vein and internal thoracic artery graft patency by coronary system. Ann Thorac Surg. 2005;79:544-51; DOI:10.1016/j. athoracsur.2004.07.047.

18. Taggart DP, D'Amico R, Altman DG. Effect of arterial revascularisation on survival: a systematic review of studies comparing bilateral and single internal mammary arteries. Lancet. 2001;358:870-5; DOI:10.1016/ S0140-6736(01)06069-X.

19. Huang DW, Sherman BT, Tan Q, Kir J, Liu D, Bryant D, Guo Y, Stephens R, Baseler MW, Lane HC, Lempicki RA. DAVID Bioinformatics Resources: expanded annotation database and novel algorithms to better extract biology from large gene lists. Nucleic Acids Res. 2007;35:W169-75 DOI:10.1093/nar/gkm415.

20. Walter W, Sánchez-Cabo F, Ricote M. GOplot: an R package for visually combining expression data with functional analysis: Fig. 1. Bioinformatics. 2015;31:2912-4; DOI:10.1093/bioinformatics/btv300.

21. Tiso N, Majetti M, Stanchi F, Rampazzo A, Zimbello R, Nava A, Daniel GA. Fine Mapping and Genomic Structure of ACTN2, the Human Gene Coding for the Sarcomeric Isoform of $\alpha$-Actinin-2, Expressed in Skeletal and Cardiac Muscle. Biochem Biophys Res Commun. 1999;265:256-9; DOI:10.1006/bbrc.1999.1661.

22. Bagnall RD, Molloy LK, Kalman JM, Semsarian C. Exome sequencing identifies a mutation in the ACTN2 gene in a family with idiopathic ventricular fibrillation, left ventricular noncompaction, and sudden death. BMC Med Genet. 2014;15:99; DOI:10.1186/s12881-014-0099-0.

23. Chiu C, Bagnall RD, Ingles J, Yeates L, Kennerson M, Donald JA, Jormakka M, Lind JM, Semsarian C. Mutations in Alpha-Actinin-2 Cause Hypertrophic Cardiomyopathy. J Am Coll Cardiol. 2010;55:1127-35; DOI:10.1016/j.jacc.2009.11.016.

24. Fan L-L, Huang H, Jin J-Y, Li J-J, Chen Y-Q, Xiang R. Whole-Exome Sequencing Identifies a Novel Mutation (p.L320R) of Alpha-Actinin 2 in a Chinese Family with Dilated Cardiomyopathy and Ventricular Tachycardia. Cytogenet Genome Res. 2019;157:148-52; DOI:10.1159/000496077. 
25. Haywood NJ, Wolny M, Rogers B, Trinh CH, Shuping Y, Edwards TA Peckham M. Hypertrophic cardiomyopathy mutations in the calponin -homology domain of ACTN2 affect actin binding and cardiomyocyte Z-disc incorporation. Biochem J. 2016;473:2485-93; DOI:10.1042/ BCJ20160421.

26. Liu $\mathrm{W}$, Zhao $\mathrm{Y}, \mathrm{Wu}$ J. Gene expression profile analysis of the progression of carotid atherosclerotic plaques. Mol Med Rep. 2018;17:5789-95; DOI:10.3892/mmr.2018.8575.

27. Notarnicola C, Rouleau C, Le Guen L, Virsolvy A, Richard S, Faure S, De Santa Barbara P. The RNA-binding protein RBPMS2 regulates development of gastrointestinal smooth muscle. Gastroenterology. 2012;143:687-697.e9; DOI:10.1053/j.gastro.2012.05.047

28. Akerberg AA, Burns CE, Burns CG. Exploring the Activities of RBPMS Proteins in Myocardial Biology. Pediatr Cardiol. 2019;40:1410-8, DOI:10.1007/s00246-019-02180-6

29. de Bruin RG, Rabelink TJ, van Zonneveld AJ, van der Veer EP. Emerging roles for RNA-binding proteins as effectors and regulators of cardiovascular disease. Eur Heart J. 2017;38:1380-8; DOI:10.1093/eurheartj/ ehw567.

30. Bonta PI, Pols TWH, de Vries CJM. NR4A Nuclear Receptors in Atherosclerosis and Vein-Graft Disease. Trends Cardiovasc Med. 2007;17:10511; DOI:10.1016/j.tcm.2007.02.001.

31. Martínez-González J, Rius J, Castelló A, Cases-Langhoff C, Badimon L. Neuron-derived orphan receptor-1 (NOR-1) modulates vascular smooth muscle cell proliferation. Circ Res. 2003;92:96-103; DOI:10.1161/01. ES.0000050921.53008.47.

32. Mirchi LF, Chylíková B, Janků M, Šeda O, Liška F. Transcriptomic analysis of left ventricle myocardium in an SHR congenic line with ameliorated cardiac fibrosis. Physiol Res. 2019;68:747-55; DOI:10.33549/ physiolres.934127.

33. Takahashi H, Nomiyama T, Terawaki Y, Kawanami T, Hamaguchi Y, Tanaka T, Tanabe M, Bruemmer D, Yanase T. GLP-1 receptor agonist exendin-4 Attenuates NR4A Orphan Nuclear Receptor NOR1 expression in vascular smooth muscle cells. J Atheroscler Thromb. 2019;26:183-97; DOI:10.5551/jat.43414

34. Martí-Pàmies I, Cañes L, Alonso J, Rodríguez C, Martínez-González J. The nuclear receptor NOR-1/NR4A3 regulates the multifunctional glycoprotein vitronectin in human vascular smooth muscle cells. FASEB 2017;31:4588-99; DOI:10.1096/fj.201700136RR.

35. Calvayrac 0, Rodríguez-Calvo R, Martí-Pamies I, Alonso J, Ferrán B, Aguiló S, Crespo J, Rodríguez-Sinovas A, Rodríguez C, Martínez-González J. NOR-1 modulates the inflammatory response of vascular smooth muscle cells by preventing NFKB activation. J Mol Cell Cardiol. 2015;80:34-44; DOI:10.1016/j.yjmcc.2014.12.015.

36. Dehghani-Samani A, Madreseh-Ghahfarokhi S, Dehghani-Samani A. Mutations of voltage-gated ionic channels and risk of severe cardiac arrhythmias. Acta Cardiol Sin. 2019;35:99-110; DOI:10.6515/ ACS.201903_35(2).20181028A.

37. Bonnet S, Paulin R, Sutendra G, Dromparis P, Roy M, Watson KO, Nagendran J, Haromy A, Dyck JRB, Michelakis ED. Dehydroepiandrosterone reverses systemic vascular remodeling through the inhibition of the Akt/GSK3- $\beta$ /NFAT Axis. Circulation. 2009;120:1231-40; DOI:10.1161/ CIRCULATIONAHA.109.848911.

38. Fancher IS, Butcher JT, Brooks SD, Rottgen TS, Skaff PR, Frisbee JC, Dick GM. Diphenyl Phosphine Oxide-1-Sensitive K+ Channels Contribute to the Vascular Tone and Reactivity of Resistance Arteries From Brain and Skeletal Muscle. Microcirculation. 2015;22:315-25; DOI:10.1111/ micc.12201.

39. Goodwill AG, Noblet JN, Sassoon D, Fu L, Kassab GS, Schepers L, Herring BP, Rottgen TS, Tune JD, Dick GM. Critical contribution of KV1 channels to the regulation of coronary blood flow. Basic Res Cardiol. 2016;111:56; DOI:10.1007/s00395-016-0575-0.

40. Barrese V, Cidad P, Yeung SY, López-López JR, McNeish AJ, Ohya S, Pérez-García MT, Greenwood IA. Proliferative role of Kv11 channels in murine arteries. Front Physiol. 2017;8:500; DOI:10.3389/fphys.2017.00500

41. Nori S, Fumagalli L, Bo X, Bogdanov Y, Burnstock G. Coexpression of mRNAs for P2X1, P2X2 and P2X4 receptors in rat vascular smooth muscle: an in situ hybridization and RT-PCR study. J Vasc Res. 1998;35:179-85; DOI:10.1159/000025582

42. Hennigs JK, Lüneburg N, Stage A, Schmitz M, Körbelin J, Harbaum L, Matuszcak C, Mienert J, Bokemeyer C, Böger RH, Kiefmann R, Klose H. The P2-receptor-mediated $\mathrm{Ca} 2+$ signalosome of the human pulmonary endothelium - implications for pulmonary arterial hypertension. Purinergic Signal. 2019;15:299-311; DOI:10.1007/s11302-019-09674-1.

43. Patel JJ, Bourne LE, Millán JL, Arnett TR, MacRae VE, Wheeler-Jones CPD, Orriss IR. Inhibition of vascular smooth muscle cell calcification by ATP analogues. Purinergic Signal. 2019;15:315-26; DOI:10.1007/ s11302-019-09672-3.
44. Avila-Smirnow D, Gueneau L, Batonnet-Pichon S, Delort F, Bécane HM, Claeys K, Beuvin M, Goudeau B, Jais JP, Nelson I, Richard P, Ben Yaou R, Romero NB, Wahbi K, Mathis S, Voit T, Furst D, van der Ven P, Gil R, Vicart P, Fardeau M, Bonne G, Behin A. Cardiac arrhythmia and late-onse muscle weakness caused by a myofibrillar myopathy with unusual histopathological features due to a novel missense mutation in FLNC. Rev Neurol (Paris). 2016;172:594-606; DOI:10.1016/j.neurol.2016.07.017. 